\title{
The SU(3) PHASe transitions in THe PRESENCE OF LIGHT DYNAMICAL QUARKS
}

\author{
R V GAVAI \\ Department of Physics, Brookhaven Nattonal Laboratory, Upton, Long Island, NY 11973, USA \\ F KARSCH \\ Department of Physics, Untversity of Illinots at Urbana-Champaign, 1110 West Green Street, \\ Urbana, IL 61801, USA
}

Received 16 April 1985

\begin{abstract}
We investigate the deconfinement and the chiral phase transitions in QCD with 3 light dynamical flavors, using the pseudo-fermıon method Monte Carlo sımulations have been performed on a lattice of size $8^{3} \times 4$ with fermions of mass 01 and 0075 respectively $A$ rapid change from the low-temperature region of hadrons to the high-temperature quark-gluon plasma is observed in all the physical quantities studied Our detalled, high-statıstics results, however, do not show any signs of a strong first-order transition In the zero mass limit we find evidence for a chiral phase transition at $T_{\mathrm{c}} / \Lambda_{L} \simeq 183 \pm 10$
\end{abstract}

\section{Introduction}

During the past few years considerable effort and computer time has been devoted to the study of the thermodynamics of quantum chromodynamics. The lattice simulations of quenched QCD [1] have reached a stage where the qualitative features like the thermodynamics in the low- and high-temperature phases and the order of the chiral and deconfinement phase transitıons are well understood and numerically well under control. Quantitative results for the critical temperature, latent heat and in part also critıcal exponents [2] are in a good shape and there relation to contınuum parameters using the non-perturbative features of the $S U(N) \beta$-function [3] lead to results which are probably reliable on the $10 \%$ level.

In contrast to this the study of the influence of dynamical fermions on the thermodynamics of QCD is still in an exploratory stage Although the results obtained so far [4-8] look very promising, they differ even on the qualitative level and are not able to predict contınuum parameters with great confidence.

Probably one of the most interesting questions, which consequently has been addressed first in the context of dynamical fermions, is their influence on the deconfinement and chiral phase transitions. In the pure gauge sector these transitions are known to be first order [9] for SU(3). Theoretical considerations based on effective models in the strong coupling region suggest that dynamical fermions tend to weaken these phase transitions. This is what one observed in a MC simulation 
with very heavy quarks, where standard MC techniques are still applicable [10] In the light mass region, however, the results for these phase transitions were inconclusive, ranging from a rapid crossover behavior [4] or a second-order phase transition $[5,6]$ to a strong first-order transition $[7,8]$ There has even been a claim of total disappearance [11] of the phase transition, although a lack of spontaneous breakdown of the chiral symmetry at $T=0$ in the model of ref [11] makes it difficult to compare with the above mentioned results. Recently most spectacular results have been presented in this context in ref. [8] where a strong first-order chiral and deconfinement transition has been reported to persist in the entire mass range down to zero-mass fermions These results have been obtained by simulating the effect of 3 quark flavors using the pseudo-fermion algorithm. They appear to be in disagreement with pseudo-fermion results on a smaller lattice [6], and also with results obtained with Wilson fermions [5] Also microcanonical simulations gave no evidence for a strong first-order transition [4]

In this paper we report the results of our detalled study of the thermodynamics of QCD with 3 flavors of light fermions We use staggered fermions and employ the pseudo-fermion algorithm to include the effect of light quarks of mass ma $=0075$ and 01 on a $8^{3} \times 4$ lattice $A$ detalled analysis of the dependence of the results on the different parameters of the pseudo-fermion approximation scheme has been performed in order to clarify the discrepancies between the results of different groups We will present evidence which suggests that the first-order signal observed in ref. [7] is most likely due to lack of convergence in the crossover region. We do not obtain any evidence for a strong discontinuity

The paper is organized as follows In sect. 2 we present the basic finite temperature formalism and fix our notations. Sect. 3 reviews the pseudo-fermion algorithm and discusses the various approximations introduced in order to make this method useful in an actual MC simulation. Sect. 4 contains our results and a comparison with earlier results of other groups and in sect 5 we present our conclusions

\section{Lattice thermodynamics}

The formalism of thermodynamics of euclidean lattices has been discussed extensively in the literature [12]. We will review here the basic features related to the introduction of staggered fermions in the formalism.

The finite temperature partition function can be regularized by introducing a lattice of size $N_{\sigma}^{3} \times N_{\tau}$ with lattice spacing a such that the volume and temperature of the system are given by $\left(N_{\sigma} a\right)^{3}$ and $T^{-1} \equiv N_{\tau} a$ respectively. For a $\operatorname{SU}(N)$ gauge theory with staggered fermions the partition function then reads

$$
Z(\beta, V)=\int \prod_{x, \mu} \mathrm{d} U_{x, \mu} \prod_{x} \mathrm{~d} \chi_{x} \mathrm{~d} \bar{\chi}_{x} \mathrm{e}^{-S(U, \bar{x}, \chi)}
$$


with the euclidean action $S$ given by

$$
\begin{gathered}
S(U, \bar{\chi}, \chi)=S_{\mathrm{G}}(U)+S_{\mathrm{F}}(U, \bar{\chi}, \chi), \\
S_{\mathrm{G}}=\frac{2 N}{g^{2}} \sum_{x, \mu<\nu}\left[1-\frac{1}{N} \operatorname{Re} \operatorname{Tr}\left(U_{x, \mu} U_{x+\mu, \nu} U_{x+\nu, \mu}^{+} U_{x, \nu}^{+}\right)\right], \\
S_{\mathrm{F}}=m \sum_{x} \bar{\chi}_{x} \chi_{x}+\frac{1}{2} \sum_{x, \mu} \bar{\chi}_{x} \eta_{\mu}(x)\left[U_{x, \mu} \chi_{x+\mu}-U_{x-\mu, \mu}^{+} \chi_{x-\mu}\right],
\end{gathered}
$$

being the gluonic and fermionic contributions to the action The fermionic fields, $\bar{\chi}, \chi$ are single component Grassmann fields defined on the sites of the lattice They also carry a flavor index which has been suppressed in eq (24) The phase factors $\eta_{\mu}(x)$ are defined as $\eta_{\mu}(x)=(-1)^{x_{1}++x_{\mu-1}}$ The action depends on the bare quark mass $m$ and the gauge coupling $\beta \equiv 2 N / g^{2}$. After integratıng over the fermionıc fields one obtains a partition function in terms of the bosonic fields $U_{x, \mu}$ alone However, in addition one gets a highly non-local contribution from the fermion determinant

$$
Z=\int \prod_{x, \mu} \mathrm{d} U_{x, \mu}\left[\operatorname{det}\left(m^{2}-D^{2}\right)\right]^{n_{f} / 8} \mathrm{e}^{-S_{\mathrm{G}}},
$$

where $D \equiv \sum_{\mu} D^{\mu}$ and

$$
D_{x y}^{\mu}=\frac{1}{2} \eta_{\mu}(x)\left[U_{x, \mu} \delta_{y, x+\mu}-U_{y, \mu}^{+} \delta_{y, x-\mu}\right]
$$

In eq. (2.5) we have introduced the positive definite operator $Q Q^{+}$where

$$
Q \equiv m+D,
$$

whose determinant is equal to the square of det $(m+D) n_{f}$ denotes the number of flavors and is required to be a multiple of 4 for staggered fermions. Following ref. [13] we will use eq. (2.5) to simulate an arbitrary number of $n_{f}$ continuum flavors

The thermodynamics and phase structure of the quark-gluon system can now be analyzed by either looking at thermodynamic observables, which are expected to show singular behavior at the phase transition temperature, or by looking at order parameter for various global symmetries of the system.

In the following we will concentrate on an analysis of the energy density

$$
\begin{aligned}
\varepsilon & =T^{2} V^{-1} \partial \ln Z / \partial T \\
& \equiv \varepsilon_{\mathrm{G}}+\varepsilon_{\mathrm{F}}
\end{aligned}
$$

where

$$
\varepsilon_{G}=3 \beta\left(\left\langle P_{\sigma}\right\rangle-\left\langle P_{t}\right\rangle\right)
$$

with $P_{\sigma(\tau)}=1-(1 / N) \operatorname{Re} \operatorname{Tr} U_{\sigma(\tau)}$ denoting the space-space (space-time) like plaquettes. The "fermionic part" of the energy density, $\varepsilon_{\mathrm{F}}$, is given by

$$
\varepsilon_{\mathrm{F}}=\frac{1}{4} n_{f}\left\langle\operatorname{tr} D^{4}(D+m)^{-1}\right\rangle-\left\{\frac{1}{16} N n_{f}-\frac{1}{4} m\langle\bar{\chi} \chi\rangle_{T=0}\right\}
$$


The term in curly brackets in eq (2.10) comes from renormalizing the energy density by subtracting the zero temperature contributions. In the defintion of the "gluonic part", $\varepsilon_{\mathrm{G}}$, of the energy density we neglected contributions which result from the derivatives of the coupling with respect to the temperature [12] These contributions are in general expected to be small, of the order of a few percent

In the absence of fermions the gluonic part of the action, $S_{\mathrm{G}}(U)$, has in addition to its local gauge symmetry a global $Z(N)$ symmetry, due to the finiteness of the euclidean lattice in time direction and the periodic boundary conditions imposed in this direction An order parameter for the realization of this symmetry is the Polyakov line

$$
L=\frac{1}{N_{\sigma}^{3}} \sum_{x} \operatorname{Re} \operatorname{Tr}\left(\prod_{x_{4}=1}^{N_{\tau}} U_{\left(x, x_{4}\right), 4}\right) .
$$

As the Polyakov line is related to the excess free energy, $F$, of a static color source in the gluonic environment, $\langle L\rangle \sim \exp \{-F / T\}$, a non-vanishing value would indicate the appearance of a deconfined phase. In the presence of dynamical fermions the $\mathrm{Z}(N)$ symmetry of the pure gauge action is explicitly broken and thus $\langle L\rangle \neq 0$ for all temperatures The Polyakov line is thus an order parameter for a deconfinement transition only in the pure gauge sector (or equivalently for infinitely heavy fermıons). Nonetheless it is clearly of interest to study its behavior in the presence of dynamical fermions also to contrast from the corresponding behavior in the pure gauge theory

In the zero mass limit the action, eq (22), has a flavor nonsinglet axial chiral symmetry for all values of lattice spacing It can be shown to be $U\left(n_{f}\right) \times U\left(n_{f}\right)$ The order parameter to check whether this symmetry is spontaneously broken is given by

$$
\langle\bar{\psi} \psi\rangle \equiv\langle\bar{\chi} \chi\rangle=\frac{1}{4} n_{f}\left\langle\operatorname{tr}(D+m)^{-1}\right\rangle
$$

\section{Simulation of dynamical fermions}

The Grassmann nature of the fermion fields reflects itself in a highly non-local determınant once these fields have been integrated out In the past different approximation śchemes have been suggested in order to deal with this determinant Presently the pseudo-fermion algorithm [13-15] and the microcanonical method [16] are widely used and seem to be most promising In the following we will discuss in some detall the pseudo-fermion algorithm and the approximations involved when implementing it in a MC simulation in order to make this method useful.

After integratıng over the fermion fields $\chi, \bar{X}$ the partition function reads

with

$$
Z=\int \prod_{x, \mu} \mathrm{d} U_{x, \mu} \operatorname{det} Q \mathrm{e}^{-S_{\mathrm{G}}}
$$

$$
\operatorname{det} Q \equiv \operatorname{det}(m+D)=\left(\operatorname{det}\left(m^{2}-D^{2}\right)\right)^{1 / 2} .
$$

The basic idea of the pseudo-fermıon method is to think of this determınant not as 
resulting from an integration over Grassmann fields but resulting from an integration over scalar fields. Actually what is needed in a MC simulation, using the Metropolis method, is not the whole determinant but its change under a change of one link variable, $U_{x, \mu} \rightarrow U_{x, \mu}+\delta U_{x, \mu}$ For small enough changes $\delta U$ we find

$$
\begin{aligned}
\frac{\operatorname{det}(Q+\delta Q)}{\operatorname{det} Q} & =\operatorname{det}\left(1+Q^{-1} \delta Q\right) \\
& =1+\operatorname{Tr} Q^{-1} \delta Q+O\left(\delta U^{2}\right) .
\end{aligned}
$$

The matrix elements $Q_{x y}^{-1}$ can then be obtained from a MC simulation with scalar fields $\phi, Q_{x y}^{-1}=\left(Q^{+} Q\right)_{x z}^{-1} Q_{z y}^{+}$

$$
\left(Q^{+} Q\right)_{x y}^{-1}=\frac{1}{Z_{\phi}} \int \prod_{x} \mathrm{~d} \bar{\varphi}_{x} \mathrm{~d} \phi_{x} \bar{\phi}_{y} \phi_{x} \exp \left\{-\sum_{l m} \bar{\phi}_{l}\left(Q^{+} Q\right)_{l m} \phi_{m}\right\}
$$

The task of evaluatıng the determınant of $Q$ for every link change is thus reduced to evaluating $Q^{-1}$. A further improvement in the time taken per link update can be brought about by noting that all the relevant matrix elements of $Q^{-1}$ may be calculated before a given sweep of all the link variables and used for the entire sweep. For the errors induced by this procedure can be shown to be $\mathrm{O}\left(\delta U^{2}\right)$ and thus negligible in the approxımation used in eq. (3.3) If $N_{\mathrm{pf}}$ denotes the number of Monte Carlo iterations over $\phi$-fields to obtain $\left(Q^{+} Q\right)_{x y}^{-1}$ using eq. (3 4$)$ then it is obvious that the algorithm (and the procedure above) becomes exact in the limit $N_{\mathrm{pf}} \rightarrow \infty$ and $\delta U \rightarrow 0$ One can thus easily recognize potential sources of statistical and/or systematic errors in any practical application of this method. Choosing too small $N_{\mathrm{pf}}$ may lead to intolerable statistical errors in $Q^{-1}$ which will be carried over in subsequent link updates whereas too large a change $\delta U$ may invalidate either the expansion, Eq (3.3), or the procedure of calculatıng $Q^{-1}$ only once per update of all links or even both. Computer time requirements clearly prevent one from going to the other extremes where, in fact, the results will necessarily be more reliable. Of course, with too small a $\delta U$ one has to be cautious again The integration in eq. (3.1) over link variables runs over the entıre group space A reliable estımate of averages for any observable may thus need increasingly large number of iterations over the link variables as $\delta U \rightarrow 0$.

In the following we will analyze in detal the dependence of the results obtained for the thermodynamics of full QCD on these parameters entering in the pseudofermion algorithm In particular we will show that they have to be handled especially carefully in a region of large correlation length in order to get conclusive results on the order of phase transitions in the presence of dynamical fermions.

\section{Results}

In the following we will present our results for a MC simulation of SU(3) gauge theory with 3 flavors of staggered fermions of mass $m a=0.1$ and 0075 on a lattice 


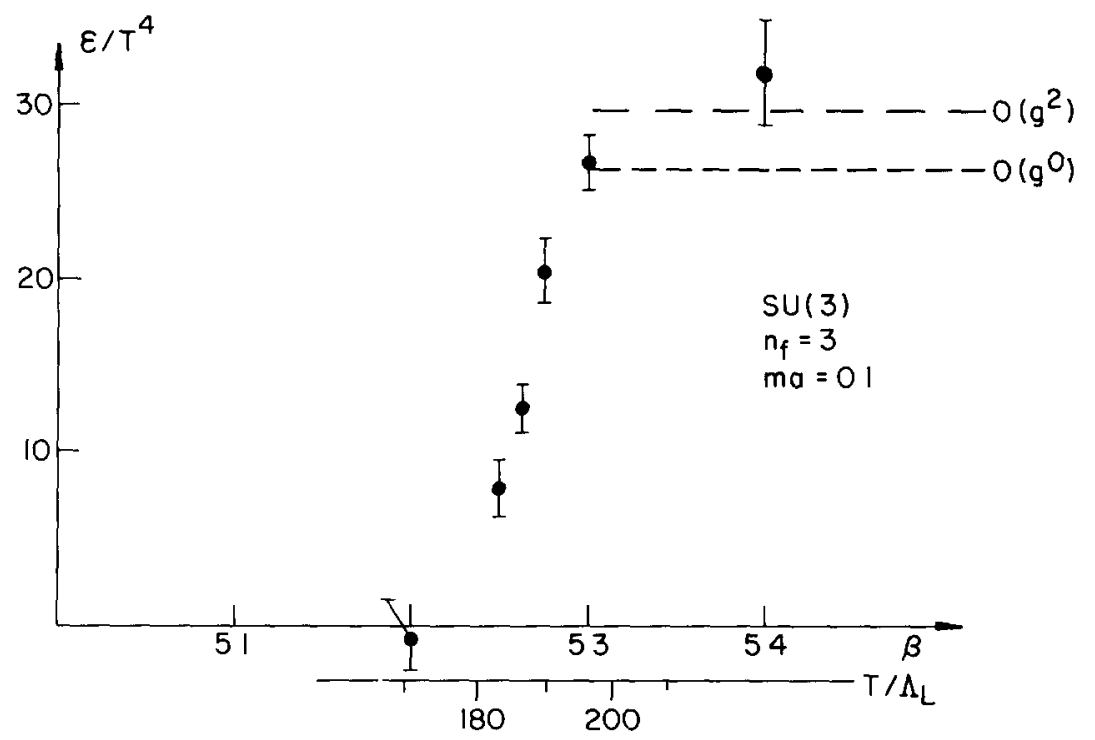

Fig 1 Energy density versus coupling $\beta$ for SU(3) with 3 flavors of mass $m a=01$ on a $8^{3} \times 4$ lattıce Also shown are the lowest order $(--)$ and $O\left(g^{2}\right)(--)$ weak coupling perturbative results The temperature scale has been obtained by assuming the validity of the asymptotic scaling relation eq (4 1)

of size $8^{3} \times 4$ The main results are based on a pseudo-fermion (pf) simulation with $N_{\text {pf }}=50$ iterations in the pf update, neglecting the first 25 for equilibration. We used a heat-bath algorithm to update the pseudo-fermions and a Metropolis algorithm with 8 hits per link for the gauge fields. The maximal change in the gauge fields allowed in a update has been adjusted such that an overall acceptance rate of $\sim 63 \%$ has been achieved. We will come back later to the question of how optimal these choices are and what effect they have on the final results.

In figs 1 and 2 we show our results for the energy density $\varepsilon$ and the Polyakov line $\langle L\rangle$ at mass $m a=0.1$ in the entire temperature range considered by us As can be seen both quantities change rapidly but seemıngly contınuously over a small coupling range $\Delta \beta=0.1\left(\Delta T / \Lambda_{L} \simeq 30\right)$. For larger couplings $\beta \equiv 6 / g^{2}$ the energy density agrees well with weak coupling results [17] At all couplings ordered and random start configurations have been analyzed to look for metastable states None have been observed Fig 3 displays evolution of the real part of the Polyakov line from a random ( $\beta=52$ quenched, thermalized configuration) start and an ordered start $\left(U_{x, \mu}=1, \forall x, \mu\right)$ at $\beta=53$ One sees that after $\approx 800$ iterations the two starts come together and thereafter yield the same value apart from statistical fluctuations. At all the couplings we studied, we observed similar behavior, the only difference being the number of iterations required to converge together, away from the critical region they decreased ${ }^{\star}$

\footnotetext{
* The slowest convergence we observed was at $\beta=525$ where the ordered start required $\sim 1500$ iterations to catch up with the random start which was equilibrated after $\sim 700$ iterations
} 


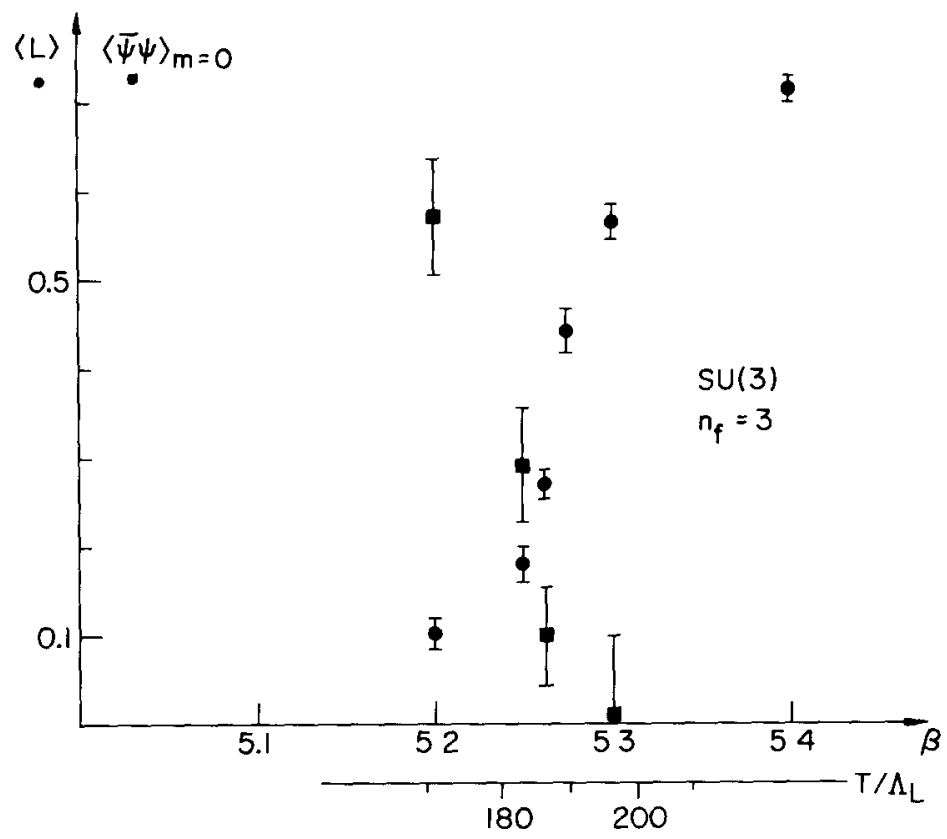

Fig 2 The Polyakov line expectation value (O) versus $\beta$ for SU(3) with 3 flavors of mass $m a=01$ on a $8^{3} \times 4$ lattice and the zero mass extrapolated chiral order parameter $(\boldsymbol{a})\langle\tilde{\psi} \psi\rangle_{m=0}$ has been obtained from a linear extrapolation of data at $m a=0075$ and 01

In fig 2 we also show the chiral order parameter $\langle\bar{\psi} \psi\rangle$ extrapolated to zero mass A linear extrapolation from our data for $m a=0.1$ and 0075 has been made to obtain these results. Clearly $\langle\bar{\psi} \psi\rangle$ vanıshes around $\beta=525$ Assuming the valıdity of the asymptotic scalıng relation

$$
a \Lambda_{L}=\exp \left\{\frac{4 \pi^{2} \beta}{33-2 n_{f}}-\frac{459-57 n_{f}}{\left(33-2 n_{f}\right)^{2}} \ln \frac{8 \pi^{2} \beta}{33-2 n_{f}}\right\}
$$

we find for the chiral transition temperature

$$
T_{\mathrm{ch}} / \Lambda_{L}=183 \pm 10 \text {. }
$$

As in the quenched approxımation, all the physıcal quantities we considered, namely $\varepsilon,\langle L\rangle$ and $\langle\bar{\psi} \psi\rangle$, exhibit a rapid change in behavior in a small interval of $\Delta \beta$ This has been a feature of previous calculations [7] too. The difference which we find is the apparent lack of discontinuity in all of them. In this respect our findings are at least qualitatively in agreement with those of ref. [6] where $6^{3} \times 2$ lattıce with $n_{f}=2$ was used and ref. [5] where a hopping parameter expansion has been used. Our results seem to indicate that the chiral phase transition is continuous contrary to what one would have expected for $n_{f}=3$ (and larger) by considering effective chiral models [18]. It may be emphasized though that all methods to obtain 


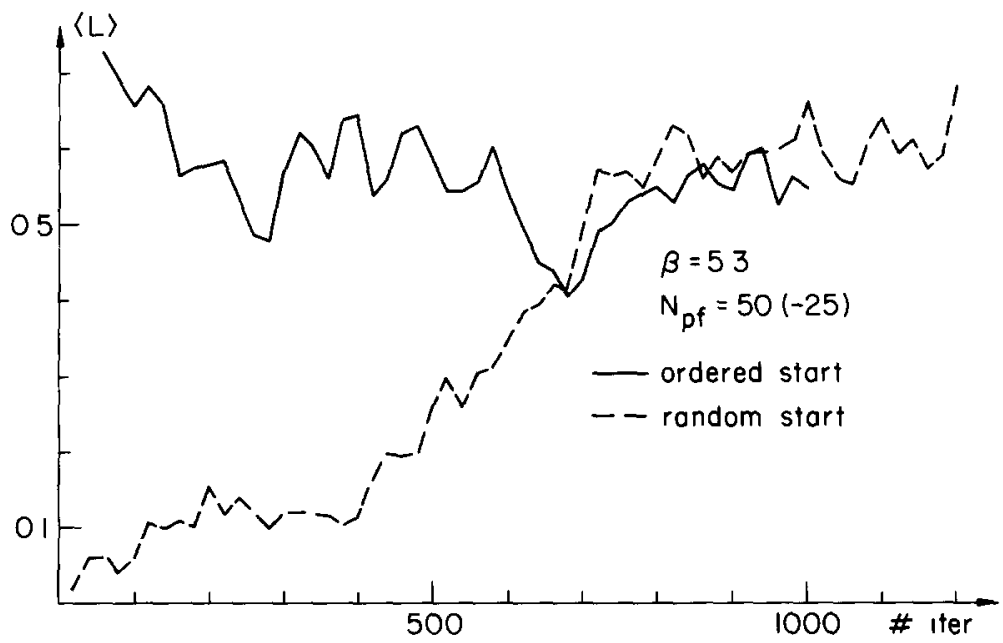

Fig 3 The Polyakov line versus number of MC iterations Shown is the evolution of $\langle L\rangle$ from random

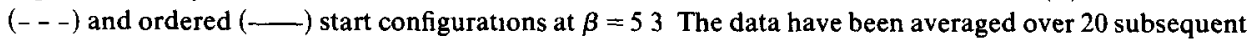
iterations

$\langle\bar{\psi} \psi\rangle_{m=0}$ from simulations on finite lattices necessarily involve extrapolations and a weak first order chiral phase transition could easily be buried in the errors of these extrapolations. Thus we certainly cannot rule out a weak fluctuation induced first-order phase transition [18].

We now turn to the discussion of the discrepancies between our present work and that of ref. [7]. Those authors also used $8^{3} \times 4$ lattice, $n_{f}=3$ and staggered fermions but they chose to use $N_{\mathrm{pf}}=24$, discarding 4 out of these to allow for equilibration and they adjusted the acceptance to be $\sim 80 \%$. Since smaller the size of $\delta U$ the greater its probability of being accepted, their acceptance rate translates into a smaller size of $\delta U$ than what we used. They presented evidence for strong first-order phase transitions: $\langle\bar{\psi} \psi\rangle$ and $\langle L\rangle$ at $m a=01$ showed discontinuities and evolution of $\langle L\rangle$ at $\beta=5.3$ (same coupling as the one used in our fig. 3) showed a two-state signal for 960 iterations Fucito et al., have recently extended this work to higher values of $m a$ and found that for all of them the first order character of the transition persists [8].

As we noted in the previous section a smaller step length $\delta U$ is clearly better. However, it may lead to problems with convergences, especially in the regions of large correlation length as in the vicinity of $\beta=5.3$ in our case; one may simply need more iterations On the other hand, our choice may have been simply too large to be acceptable for eq. (3.3) to be still valıd. In table 1 we show that the latter is most likely not the case. We compare the average plaquette values $\left(\frac{1}{3} \operatorname{Re} \operatorname{Tr} U_{\mathrm{p}}\right)$ at $\beta=5.2,5.3$ and 5.4 with those obtained by Fucito, Rebb1 and Solomon with $80 \%$ acceptance. They agree extremely well Even at $\beta=5.3$ our results are in agreement 
TABLE 1

Comparison of plaquette expectation values at different values of $\beta$ and $m a=01$

\begin{tabular}{cccc}
\hline & & $\frac{1}{3} \operatorname{Re} \operatorname{Tr} U_{\mathrm{p}}$ \\
\hline$\beta$ & $n_{f}=3$, acc $=80 \%$ & $n_{f}=3$, acc $=63 \%$ & $n_{f}=0$ \\
\hline 52 & $0481 \pm 0002$ & $04813 \pm 00008$ & $043173 \pm 000040$ \\
53 & $0528 \pm 0001$ & $05283 \pm 00008$ & $047163 \pm 000087$ \\
54 & $0545 \pm 0002$ & $05475 \pm 00010$ & 0471030 \\
\hline
\end{tabular}

The first column shows the results of ref [7] obtained with an acceptance rate of $80 \%$ The second column gives our results obtained with $63 \%$ acceptance The last column shows pure gauge theory results

with their ordered start. We take this reassuring agreement on the level of 0.001 to mean that our choice of $63 \%$ acceptance rate is at least as good as theirs. Both the works indicate that the inclusion of dynamical fermions changes the average plaquette by approximately 0.05 compared to the pure gauge values.

In order to test whether our first hypothesis about the discrepancy is correct, we made long runs at $\beta=53, m a=0.1$ starting from the same random configuration but with acceptance maintained at $\sim 53 \%, 63 \%$ and $79 \% \quad N_{\mathrm{pr}}$ was chosen to be 24 and 4 iterations were discarded so as to be able to compare with ref. [7]. Fig. 4 exhibits the results of this study. Also shown is the equilibrium value at $\beta=5.3$ obtained from the run displayed in fig. 3. One sees a clear rising trend in all the three curves. Though one needs more than 2000 iterations to be convinced that even with $79 \%$ acceptance the final result will be the same This perhaps explains why the authors of ref. [7] interpreted their results as signals for two state behavior after

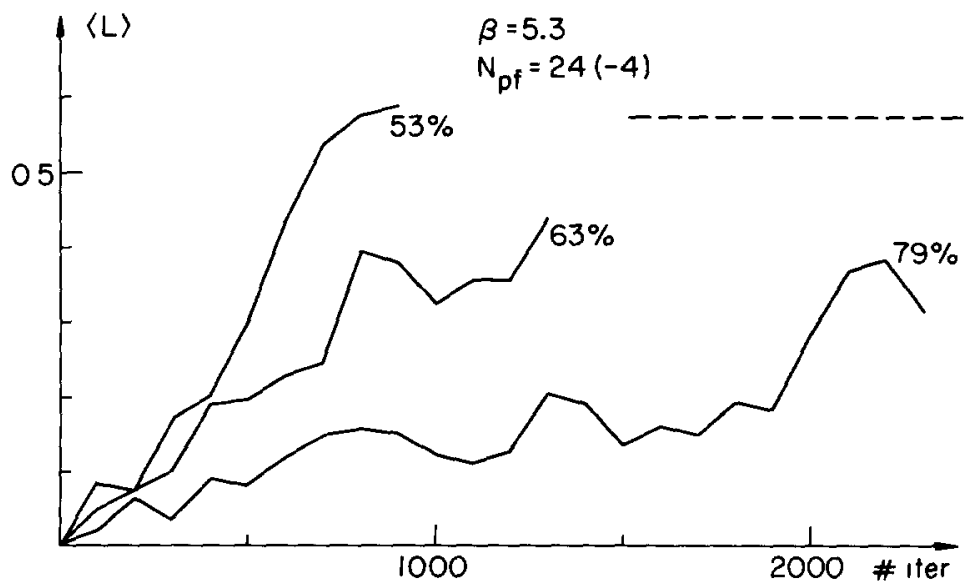

Fig 4 The Polyakov line versus number of MC iterations for vanous acceptance rates at $\beta=53$ The dashed line indicates the equilibrium value obtained in the run shown in fig 3 The data have been averaged over 50 subsequent iterations 


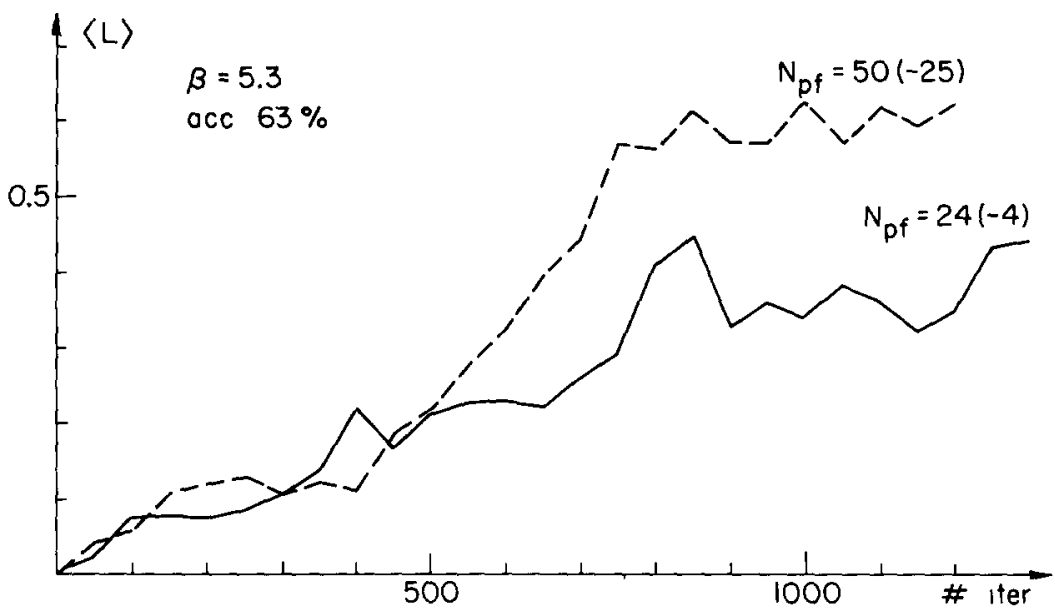

Fig 5 The Polyakov line versus number of MC iterations for different number of pseudo-fermion tterations $\left(N_{p f}\right)$ at $\beta=53$ and fixed acceptance rate of $63 \%$ The numbers in brackets denote the terations disgarded before taking averages The data have been averaged over 50 subsequent iterations

1000 iterations. In fig 5 we display the dependence on $N_{\mathrm{pf}}$. We compare at $\beta=5.3$ and $m a=0.1$ the two choices of $N_{p r}$ used by us and ref. [7]. One notices that equilibration time depends on $N_{\mathrm{pf}}$ too.

To summarize then we find that the pseudo-fermion method works rather well with comparatively small acceptance rates also Average values of physical observables tend to be quite independent of the parameters $N_{\mathrm{pr}}$ and $\delta U$, provided one makes sure that equilibrium is reached. The convergence rate appears to depend strongly on both these parameters and if one prefers to optimize for smaller $\delta U$ then extra care needs to be taken to ensure that measurements are made in equilibrium only

\section{Conclusions}

We have studied the thermodynamics of SU(3) with 3 light quark flavors. A rapid change from the low-temperature phase to the high-temperature quark-gluon plasma has been observed In the zero-mass limit we find evidence for a chiral phase transition In view of the present data it is suggestive that the first-order phase transition present in the pure gauge sector of the theory weakens and may disappear at some critical mass value. MC simulations on a $6^{3} \times 2$ lattice [19] indicate that this happens around $m_{\mathrm{c}} / T_{\mathrm{c}} \leqslant 24$ This is considerably smaller than what has been estımated earlier from a large mass approximation [10] However, in total we believe a generic phase diagram like the one shown in fig 6 may be emerging out of these MC simulations for SU(3) with 3 flavors To support this picture it certainly would be interesting to find out whether the second-order endpoint (A) exists in this phase diagram also on larger lattices, to determine $m_{c}$ at that point and confirm the 


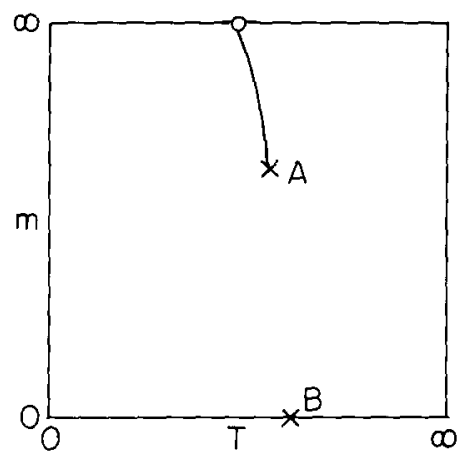

Fig 6 Generic phase diagram for SU(3) with 3 flavors The circle on the $m=\infty$ line indicates the first order phase transition in the pure gauge sector from which a line of first order transitions emerges ending in a second order transition at the point $A$ The point $B$ indicates the second order chiral transition at $m=0$

universality of the ratio $m_{\mathrm{c}} / T_{\mathrm{c}}$. Whether the rapid crossover behavior seen for light quark masses between $m_{\mathrm{c}} a$ and the chiral transition at $m a=0$ is just a remnant of these phase transitions or indicates a line of second-order transitions connecting the points at $A$ and $B$ remains unclear on the basis of the present data Our understanding of the QCD phase diagram in the presence of fermions is, however, still incomplete. Surprises may also show up by analyzing in more detail the flavor dependence of the phase diagram [20].

This work was supported in part by a grant of the National Science Foundation (NSF-PHY82-01948) and the US Department of Energy under contract DE-AC02$76 \mathrm{CH} 00016$.

\section{References}

[1] F Karsch, The deconfinement transition in finite temperature lattice gauge theory, preprint CERNTH 4003 (1984),

J Cleymans, R V Gaval and E Suhonen, Phys Reports to be published

[2] R V Gava1 and H Satz, Phys Lett 145B (1984) 248,

G Curci and R Tripiccione, Phys Lett 151B (1985) 145

[3] $\mathrm{K} \mathrm{C} \mathrm{Bowler} \mathrm{et} \mathrm{al,} \mathrm{Monte} \mathrm{Carlo} \mathrm{renormalization} \mathrm{group} \mathrm{studies} \mathrm{of} \mathrm{SU(3)} \mathrm{lattıce} \mathrm{gauge} \mathrm{theory,}$ Edınburgh preprint No 851335 (1985) and references thereın

[4] J Polonyi, H W Wyld, J B Kogut, J Shigemitsu and D K Sinclair, Phys Rev Lett 53 (1984) 644

[5] T Celık, J Engels and H Satz, Phys Lett 133B (1984) 427

[6] R V Gaval, M Lev and P Peterson, Phys Lett 140B (1984) 397, 149B (1984) 492

[7] F Fucito, and S Solomon, Phys Lett 140B (1984) 387,

F Fucito, C Rebbi and S Solomon, Nucl Phys B248 (1984) 615, Phys Rev D31 (1985) 1461

[8] F Fucito, R Kinney and S Solomon, CALT-68-1189 (1984)

[9] T Celik, J Engels and H Satz, Phys Lett 125B (1983) 411,

J Kogut, M Stone, H W Wyld, W R Gıbbs, J Shıgemitsu, SH Shenker and D K Sinclair, Phys Rev Lett 50 (1983) 393

[10] P Hasenfratz, F Karsch and I O Stamatescu, Phys Lett 133B (1983) 221

[11] M Fischler and R Roskies, Phys Lett 145B (1984) 99 
[12] J Engels, F Karsch, I Montvay and H Satz, Nucl Phys B205 [FS5] (1982) 545

[13] H W Hamber, E Mannarı, G Parısı and C Rebbi, Phys Lett 124B (1983) 99

[14] F Fucito, E Marinar1, G Parisi and C Rebb1, Nucl Phys B180 [FS2] (1981) 369

[15] E Marınarı, G Parısı and C Rebbı, Nucl Phys B190 [FS3] (1981) 734

[16] J Polony1 and H W Wyld, Phys Rev Lett 51 (1983) 2257

[17] U Heller and F Karsch, Nucl Phys B251 [FS13] (1985) 254, CERN preprint, CERN-TH 4078/84

[18] R D Pisarskı and F Wilczek, Phys Rev D29 (1984) 338

[19] N Attıg, R V Gavai, B Peterson and M Wolft, in preparation

[20] J B Kogut, J Polonyı, H W Wyld and D K Sinclatr, Simulations and speculations on gauge theories with many fermions, Illinois preprint, ILL-(TH)-85-15 (1985) 